\title{
A Novel Statistical Spring-Bead Based Network Model for Self-Sensing Smart Polymer Materials
}

\author{
Jinjun Zhang ${ }^{1}$, Bonsung Koo ${ }^{1}$, Yingtao Liu ${ }^{2}$, Jin Zou ${ }^{1}$, Aditi Chattopadhyay ${ }^{1}$ and Lenore Dai ${ }^{1}$ \\ ${ }^{1}$ School for Engineering of Matter, Transport, and Energy, Arizona State University, Tempe, AZ, USA 85287 \\ ${ }^{2}$ School of Aerospace and Mechanical Engineering, University of Oklahoma, Norman, OK, USA 73019
}

This paper presents a multiscale modeling approach to simulate the self-sensing behavior of a load sensitive smart polymer material. A statistical spring-bead based network model is developed to bridge the molecular dynamics simulations at the nanoscale and the finite element model at the macroscale. Parametric studies are conducted on the developed network model to investigate the effects of the thermoset crosslinking degree on mechanical response of the self-sensing material. The comparison between experimental and simulation results shows that the multiscale framework is able to capture the global mechanical response with adequate accuracy and the network model is also capable of simulating the self-sensing phenomenon of the smart polymer. Finally, the molecular dynamics simulation and network model based simulation are implemented to evaluate damage initiation in the self-sensing material under monotonic loading.

\section{Nomenclature}

$\begin{array}{ll}I & =\text { fluorescence intensity density } \\ L_{i} & =\text { original length of the } i \text { th spring } \\ \Delta L_{i} & =\text { variation of the spring length } \\ N & =\text { number of springs in this subzone } \\ N_{\text {Actual }} & =\text { number of actual potential crosslinked covalent bonds } \\ N_{\text {Ideal }} & =\text { number of all potential crosslinked covalent bonds } \\ \varepsilon & =\text { loading strain } \\ \varepsilon_{i} & =\text { equivalent strain in the } i \text { th spring } \\ \bar{\varepsilon} & =\text { average equivalent strain in the bead-centered subzone } \\ \theta & =\text { crosslinking degree }\end{array}$

Keywords: spring-bead model, network model, statistical distribution of crosslinking degree, self-sensing smart material

\section{Introduction}

$\mathrm{L}$ oad sensitive materials that exhibit specific chemical reactions upon external loading (also known as mechanophores) have received considerable attention in recent years. ${ }^{[1-4]}$ Mechanophores, which are covalently linked into polymers, is a result of the transmission of external forces at the macroscale to the debonding of covalent links at the molecular level. ${ }^{[4,5]}$ Therefore, itprovides a novel means for designing smart materials with self-sensing capability. Cho et al. ${ }^{[6,7]}$ have developed a cyclobutane-based mechanophore comprised of carbon-carbon covalent bonds that transform into cinnamate groups and emit visible fluorescence under external loading. Zou et al. ${ }^{[8]}$ have developed a novel self-sensing technique in which Tris-(Cinnamoyl oxymethyl)-Ethane (TCE) is embedded as the cyclobutane-based mechanophore material within a polymer matrix. In this study, cyclobutane-containing crosslinked polymers are incorporated into an epoxy matrix and the effect on their mechanical properties are examined, followed by a demonstration of early damage detection through mechanically induced fluorescence. This experimental research has shown significant promise in developing mechanophore embedded polymer materials that 
can be used to detect damage initiation in polymeric composite materials. ${ }^{[8]}$ However, the discovery of new mechanophores (i.e., mechanochemically reactive units) is currently time-consuming, expensive, low-yielding, and a process that is not easily scaled to large quantities. High fidelity modeling techniques are necessary to understand the physics of mechanophores. A will developed modeling approach can simulate the multiscale response of these "smart" polymers, which will be useful to design new polymers with mechanophores as well as apply the available materials for large scale engineering applications.

In epoxy based polymers, the crosslinked bond clusters between the resin and the hardener molecules generate a large-scale network structure. The heterogeneous crosslinked network at the microscale has significant impact on the local mechanical properties. ${ }^{[9-11]}$ In order to design and synthesize polymers with multifunctional capabilities, it is important to develop a fundamental understanding of the effects of microscale variability on material properties and response, such as the Young's modulus, heterogeneous stress/strain distribution and damage initiation mechanisms. Currently, there are numerous approaches to the modeling of polymeric systems integrated with nanoparticles; they include homogenization techniques, the finite element (FE) method, molecular dynamics (MD) simulation, Monte Carlo (MC) simulation, and the Mori-Tanaka approach. ${ }^{[12-14]}$ MD simulations and FE analysis are two of the most widely used methods for understanding the material behavior of epoxy-based systems at the nano and microscales. MD simulation is capable of effectively capturing the heterogeneous material properties of a polymer that are influenced by its crosslinking degree. ${ }^{[9-11]}$ Similarly, Fan et al. ${ }^{[1]}$ performed MD simulations for characterizing material properties of a crosslinked epoxy resin compound. Using this model, they were able to capture linear thermal expansion coefficients and Young's modulus of the material. MD simulations have also been used to investigate the strength and molecular mechanisms of adhesion between an inorganic substrate and a cured epoxy resin. ${ }^{[15]}$ In this model, the crosslink density and corresponding material properties of the crosslinked system were accurately estimated. However, the high computational cost associated with large-scale MD simulations is a major restriction and often limits its application beyond the nanoscale.

FE modeling has been a common method to model material performance, primarily because it is computationally efficient. ${ }^{[16-18]}$ Onck et al. developed a two-dimensional model to study the strain stiffening of crosslinked filamentous polymer. ${ }^{[19]}$ The FE method was used to discretize the filament with Euler-Bernoulli beam elements that accounted for stretching and bending. Jiang et al. performed a parametric study using FE analysis to compute the material properties and scratch behavior of a crosslinked polymer. ${ }^{[20]}$ The simulation results in this work corresponded well with the mechanical properties obtained from the experiments. Chen and Lagoudas developed a constitutive theory for polymers in which the crosslinking density was considered as the major influence on the mechanical behavior. ${ }^{[21]}$ This model has been implemented and extended by other researchers to study thermoelasticity, viscoelasticity, and nonequilibrium relaxation. ${ }^{[22-24]}$ While the FE method provides advantages in high simulation efficiency to model polymers, it cannot be used to model heterogeneous microstructure because the definition of discontinuous material properties is often infeasible in FE approach. Therefore, new techniques with improved computational efficiency and high accuracy are desired for investigating the material behavior of polymers at the microscale.

In addition to FE and MD models, another method commonly adopted is the discrete material techniques, such as the spring-bead model, have been developed to study polymer material. ${ }^{[25-27]}$ Underhill and Doyle developed a new method for generating coarse-grained models of polymers. ${ }^{[28]}$ To represent the larger scale material behavior of polymers, spring-bead chains have been further crosslinked to generate a network model. ${ }^{\text {[29] }}$ A spring-bead based network model that bridges nanoscale information to higher length scales, has been developed to model the structural epoxy embedded with TCE monomer. ${ }^{[8,30]}$ The material was partitioned into discrete mass beads, which were then linked using linear springs at the microscale. By integrating multiple spring-bead models, a network model was constructed to represent the material properties at the mesoscale. A series of MD simulations were performed initially to define the spring stiffness in the statistical network model using a well-known MD simulation tool called LAMMPS (Large-scale Atomic/Molecular Massively Parallel Simulator), and a force field for general organic materials called MMFF (Merck molecular force field). ${ }^{[31,32]}$ Compared with the MD model, only the equivalent mechanical responses that significantly influence the damage initiation was captured in the spring-bead based network model. Additional material properties, such as thermal and chemical properties, were omitted in order to improve simulation efficiency. The developed multiscale methodology was demonstrated as being computationally efficient and able to provide a possible means to bridge various length scales (from $10 \mathrm{~nm}$ in MD simulation to $10 \mathrm{~mm}$ in FE model) without significant loss of accuracy. In this paper, the statistical network model is further investigated to understand the effects of the model parameters on material response and damage in the TCE embedded polymer. The model is also used to develop a relationship between mechanical strain and experimentally observed fluorescence, with damage growth in the smart polymer under multiaxial loading. 
In a crosslinked network model, material performance is affected by parameters, such as crosslinking degree, chain flexibility and chain lengths. ${ }^{[33-35]}$ Among all variabilities, crosslinking degree, which represents the degree of microscopic crosslinked covalent bonds, is considered as one of the major effects on the mechanical properties of polymers. ${ }^{[33,36-38]}$ Urbaczewski-Espuche et al. developed a crosslinked network model and studied the influence of the crosslinking degree on the mechanical properties of epoxy. ${ }^{[39]}$ The results showed that the mechanical properties including Young's modulus, ultrasonic modulus, thermomechanical relaxation, and plastic behaviors are all affected by crosslinking degree. Svaneborg et al. investigated the effect of microscopic disorder on the mechanical response of network models with the same crosslinking degree. ${ }^{[40]}$ They concluded that the heterogeneities in the randomly generated crosslinked network models cause significant differences in the localization of monomers. An important objective of this work was to understand the microscopic performance of smart polymeric material through a parametric study of the network model. A series of simulations were performed to compare the effects of the crosslinking degree on the mechanical response, such as strain distribution. The knowledge obtained was important for enhancing material performance, and for methods required for controlling crosslinking degree during the manufacturing process. In addition, this study led to a preliminary investigation for evaluating damage accumulation and growth under monotonic loading.

In this paper a multiscale modeling approach is developed to simulate the mechanophore reactions of an epoxy based smart polymer system bridging between the nanoscale and micro/macroscales. In Section II, the developed spring-bead network model is introduced in details. The parametric study of the spring-bead model is discussed in Section III. The multiscale network model is presented in Section IV. The comparison of the experimental validation of the simulated damage is shown in Section V.

\section{Development of spring-bead based network model}

Zhang and Chattopadhyay recently developed a novel multiscale model that can characterize the material behavior of the crosslinked polymer. ${ }^{[30]}$ The schematic of the multiscale approach adopted in this work is shown in figure 1. A brief description of the model is presented here as a comprehensive overview. At the microscale, a spring-bead model was used to capture the mechanical response of a crosslinked cluster of polymer chains. MD simulations were performed to provide local material properties represented by springs. At the mesoscale, the network model consisting of a series of springs and beads was developed to represent larger scale crosslinked polymer chains and for capturing the mechanical responses. Mechanical equivalence optimization was then implemented to identify the relationship between the crosslinking degree and its corresponding representative spring stiffness in the network model. This network model was capable of bridging the high accuracy MD model at the microscale and the computationally efficient FE model at the macroscale, serving as a simplified mechanical equivalent of MD models with significant reduction in computational effort.

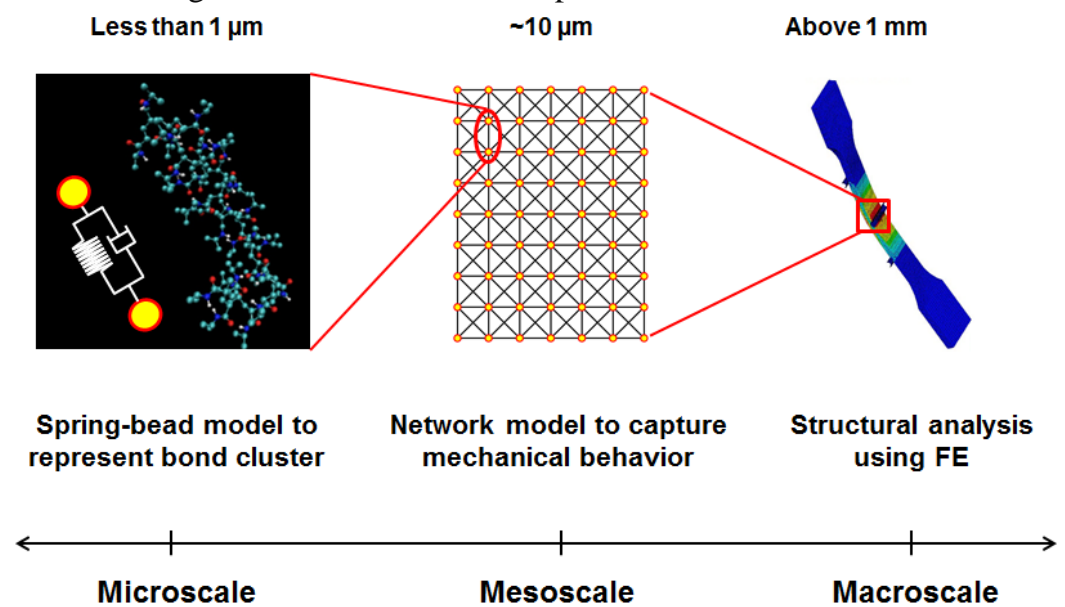

Figure 1. Modeling of smart polymer materials at the microscale, mesoscale, and macroscale.

The multiscale approach developed in this paper was as follows: First, the material behavior of the heterogeneous crosslinked microstructure was determined using MD simulation. Di-Glycidyl Ether of Bisphenol F (DGEBF) and Di-Ethylene Tri-Amine (DETA), respectively, were used for the molecular structures of the epoxy resin and the hardener that form the polymer. In order to capture the variability in the crosslinking degree and the mechanical properties of the polymer system, a statistical approach was used, whereby a representative volume 
element (RVE) model was constructed with molecules of the resin, hardener, and smart material, which were randomly distributed in the initial configuration (see figure 2). The weight percentages of the components in this smart polymer were $70.9 \%$ for DEGBF, $19.1 \%$ for DETA, and $10.0 \%$ for TCE. In the crosslinked polymer material, the crosslinking degree, which is defined as the percentage of actual crosslinked covalent bonds over all potential crosslinked bonds, significantly influences the mechanical properties at the microscale. It is important to accurately capture the crosslinking degree using MD simulations. The crosslinking degree can be defined in Eq. (1):

$$
\theta \%=\frac{N_{\text {Actual }}}{N_{\text {Ideal }}}
$$

where $\theta$ is the crosslinking degree and $N_{\text {Actual }}$ and $N_{\text {Ideal }}$ are the number of the actual and all potential crosslinked covalent bonds in the same zone at the microscale, respectively. In this approach, a total of $500 \mathrm{MD}$ simulations were performed to represent the crosslinking degree of the target system, each with the same molecules (42,350 atoms in each RVE) but randomly generated initial configuration (different initial positions). The size of RVE models was $14 \mathrm{~nm} \times 14 \mathrm{~nm} \times 14 \mathrm{~nm}$. After performing energy minimization based on the conjugate gradient algorithm to avoid undesired repulsive forces between atoms, iso-baric/iso-thermal (NPT) ensemble simulations was conducted to equilibrate each RVE at standard room condition (300K and 1atm). Periodic boundary conditions (PBC) were applied to the boundaries of the RVE. The equilibrated RVE was cured using a bond formation command in LAMMPS with a cut-off radius of 4.0 Angstroms; this cut-off radius was selected because it is more than twice the length of a carbon-nitrogen covalent bond. Based on the simulation results there was a quasi-normal distribution for the crosslinking degrees, of which the range was between $40 \%$ and $65 \%$ and the average value was $52.72 \%$ (see figure 3). MD simulations were further performed on the crosslinked polymeric systems to obtain the mechanical responses (tensile, compressive, and shear moduli) associated with variable crosslinking degrees. The moduli can be described as functions of the crosslinking degree. These results were used to construct the springbead based network model.

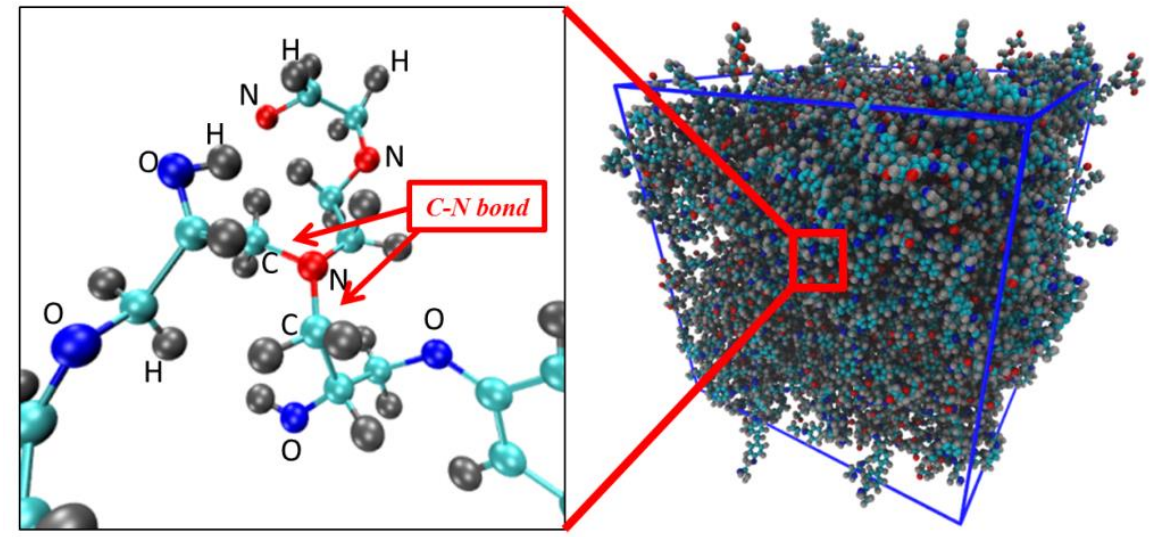

Figure 2. RVE model of smart polymer; comprises epoxy resin, hardener, and smart material (grey, cyan, blue, red sphere represent hydrogen, carbon, oxygen, and nitrogen atoms, respectively).

The next step was to develop a spring-bead based network model to characterize the material behavior of a crosslinked cluster of polymer chains with inputs from MD simulations. In the spring-bead model (see figure 1) each of the two beads served as half of the bond cluster mass; the linear spring was used to represent the elastic mechanical response (tensile and compressive) of the bond cluster. Variable spring stiffness (ratio of force to relative deformation) was used to represent bond clusters with different crosslinking degrees. To represent larger scale material behavior, a network model consisting of a series of spring-bead models was developed (see figure 1). A set of network models with different combination methods was generated. The "8 neighbor based" network model was then found to be optimal having structural stability, good representation of isotropic property, and high computational efficiency. ${ }^{[30]}$ Therefore, in this paper, all parametric studies and analyses were performed using the 8 neighbor based network model.

Mechanical equivalence optimization was implemented to integrate the mechanical responses from the MD simulations and the network model. A multiobjective optimization technique was used to establish a relation between the local crosslinking degree and the associated representative spring stiffness in the network model. 
Further details of the multiscale modeling approach of the smart polymer material can be found in the authors' previous work. ${ }^{[30]}$

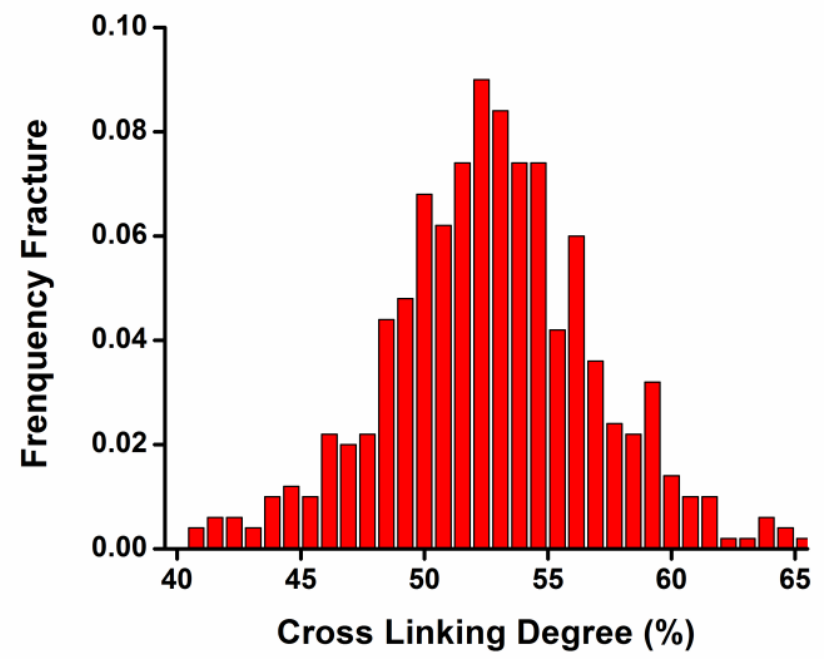

Figure 3. Statistical distribution of crossing-linking degrees based on MD simulation.

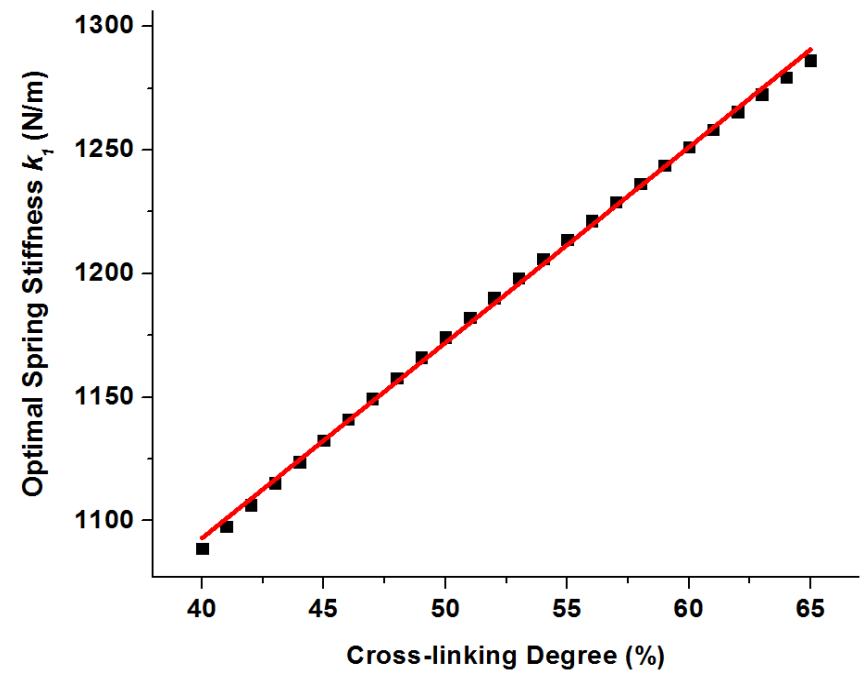

Figure 4. Polynomial fitting curve of spring stiffness based on optimization results.

\section{Parametric study of spring-bead based network model}

\section{A. Homogeneous and heterogeneous distribution of crosslinking degree}

In this section, a parametric study of the network model is conducted to understand the effect of the most critical microscopic variable, the crosslinking degree, which influences the distribution of stress, strain, strain energy, etc. The network model was constructed by discrete beads and crosslinked springs instead of continuous material. Therefore, the traditional strain concept was not applicable. A new definition of strain is required prior to the parametric study and analyses of the network model. In traditional continuous materials strain is defined as a geometrical measure of deformation representing the relative displacement between particles in a material body. In the spring-bead based network model the "equivalent strain" in one spring is defined as the deformation in terms of original and relative length of this spring, as shown in Eq. (2): 


$$
\varepsilon_{i}=\left\|\frac{\Delta L_{i}}{L_{i}}\right\|
$$

where $L_{i}$ is the original length of the $i$ th spring, $\Delta L_{i}$ is the variation of the spring length, and $\varepsilon_{i}$ is the equivalent strain in the spring. In the 8 neighbor based network model, each bead is linked with eight neighbor beads by springs (see figure 1 and figure 5). The black solid lines and yellow circles in figure 5 represent the springs and beads, respectively. To evaluate material deformation in a zone, the average equivalent strain must be defined in terms of relative springs within the same zone. The average equivalent strain in the bead-centered area (the square zone with grey color in figure 5) is defined as the averaged value of all strain in the eight neighbor springs, which are then lin to the centered bead. For the 8 neighbor based network model, the average equivalent strain in the grey area is defined in Eq. (3):

$$
\bar{\varepsilon}=\frac{1}{N} \cdot \sum_{i=1}^{N} \varepsilon_{i}
$$

where $\varepsilon_{i}$ is the equivalent strain in the $i$ th spring, $\bar{\varepsilon}$ is the average equivalent strain in the bead-centered subzone, and $N$ is the number of springs in this subzone. $N=8$ for the 8 neighbor based network model.

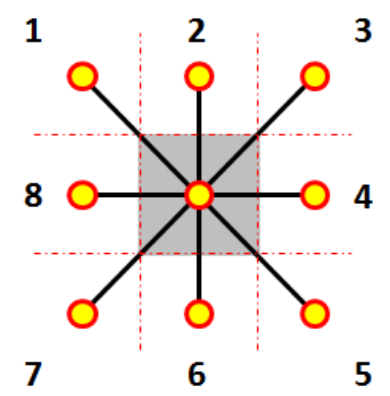

Figure 5. Beads and springs in the 8 neighbor based network model.

The influence of heterogeneous distribution of the crosslinking degree on the mechanical response is studied. A set of four network models (size: $1 \mathrm{~mm} \times 1 \mathrm{~mm})$ were generated. There are $100(10 \times 10)$ subzones in each of the four network models. As shown in figure 6(a), the schematic network model consists of two subzones (A and B) representing two different crosslinking degrees. The degree of heterogeneity, which is defined as the difference in crosslinking degree between neighbor subzones, for all four network models is present in Table 1 . It can be seen that although the average crosslinking degree value remains the same value $(53 \%)$, the degree of heterogeneity increases gradually from model 1 to model 4 . Note that the crosslinking degree value is directly related to the material's properties, which are represented by spring stiffness. The spring stiffness in subzones A and B, for all four models, is shown in figure 6(b). The increasing difference in spring stiffness between neighbor subzones also indicates that the material properties change from homogeneous to heterogeneous.

Table 1. Crosslinking degree and degree of heterogeneity in the four network models

\begin{tabular}{|l|c|c|c|}
\hline & crosslinking degree in A & crosslinking degree in B & heterogeneous degree \\
\hline Model 1 & $53 \%$ & $53 \%$ & 0 \\
\hline Model 2 & $56 \%$ & $50 \%$ & $6 \%$ \\
\hline Model 3 & $59 \%$ & $47 \%$ & $12 \%$ \\
\hline Model 4 & $62 \%$ & $44 \%$ & $18 \%$ \\
\hline
\end{tabular}




\begin{tabular}{|l|l|l|l|l|l|l|l|l|l|}
\hline A & B & A & B & A & B & A & B & A & B \\
\hline B & A & B & A & B & A & B & A & B & A \\
\hline A & B & A & B & A & B & A & B & A & B \\
\hline B & A & B & A & B & A & B & A & B & A \\
\hline A & B & A & B & A & B & A & B & A & B \\
\hline B & A & B & A & B & A & B & A & B & A \\
\hline A & B & A & B & A & B & A & B & A & B \\
\hline B & A & B & A & B & A & B & A & B & A \\
\hline A & B & A & B & A & B & A & B & A & B \\
\hline B & A & B & A & B & A & B & A & B & A \\
\hline
\end{tabular}

a)

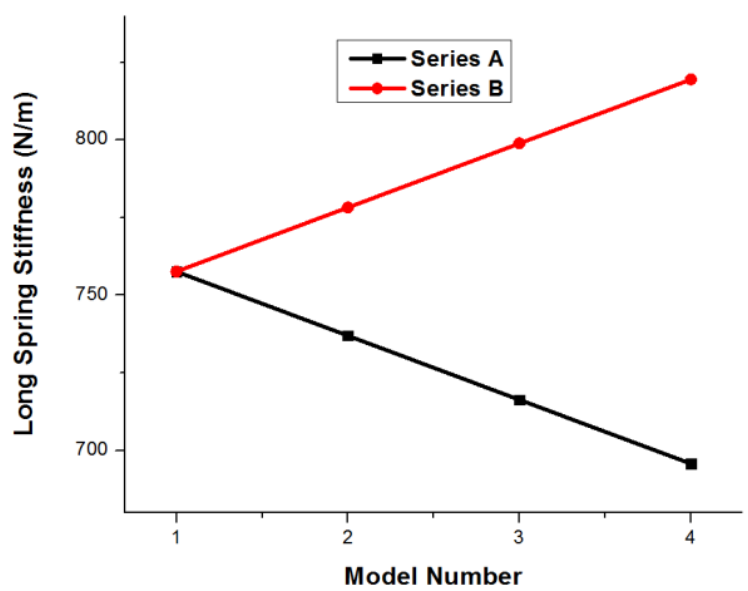

b)

Figure 6. a) Specific network model to study effect of degree of heterogeneity and b) spring stiffness in subzone A and $\mathrm{B}$ of the network model 1, 2, 3 and 4 .

Simulations were conducted using uniaxial tensile load applied to all four network models. The loading is strain controlled and the maximum loading strain is 5\%. The equivalent strain is used to evaluate the mechanical responses in the network models. The simulation results are shown in Table 2 and figure 7 . In all four models, the average equivalent strain is nearly the same. It can be observed that the difference between the maximum and the minimum equivalent strain in the network models is also influenced by the degree of heterogeneity (see Table 2). The difference increases with degree of heterogeneity (see figure 7).

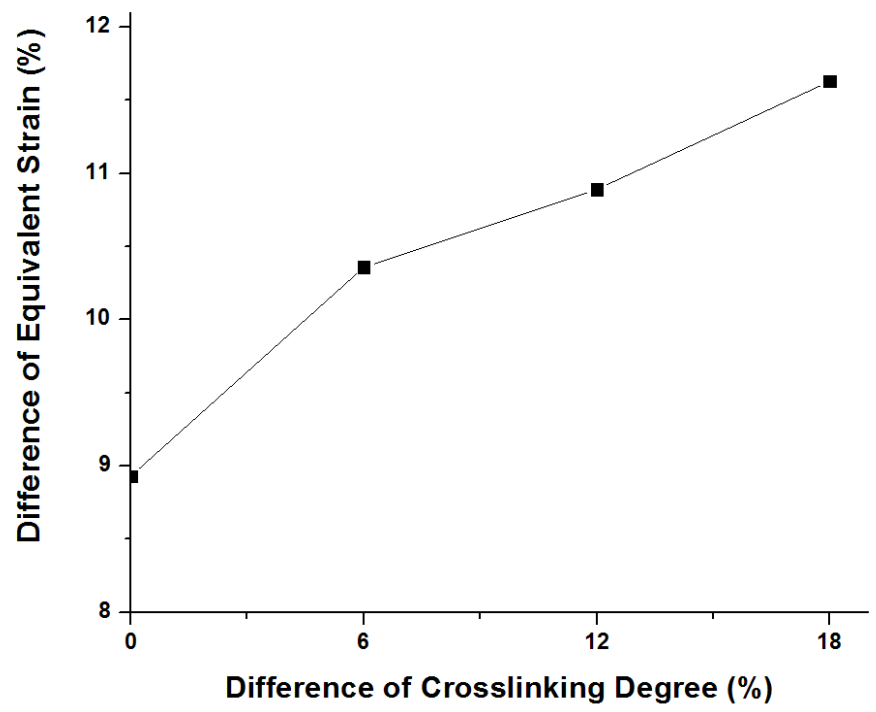

Figure 7. The difference in neighbor crosslinking degree influences the difference between maximum and minimum equivalent strain in the network model.

Thus, the parametric study involving heterogeneous crosslinking degree distribution revealed that a larger degree of heterogeneity leads to a higher difference of equivalent strain. It must be noted that in brittle materials, strain gradient plays an important role in singular or non-singular stress concentrations and local damage. ${ }^{[41]}$ It has been found that a higher local strain gradient can accelerate damage initiation. ${ }^{[42,43]}$ From the simulations and analyses of results, the increasing degree of heterogeneity can increase the strain gradient, which leads to a higher probability of damage evolution. These observations indicate that a reduction in the degree of heterogeneity can help to reduce the damage initiation. 
Table 2. Equivalent strain in network models to study degree of heterogeneity effect

\begin{tabular}{|c|c|c|c|c|}
\hline & Model 1 & Model 2 & Model 3 & Model 4 \\
\hline Average equivalent strain & 0.0339 & 0.0339 & 0.0339 & 0.0338 \\
\hline Maximum equivalent strain & 0.0358 & 0.0357 & 0.0358 & 0.0361 \\
\hline Minimum equivalent strain & 0.0326 & 0.0320 & 0.0319 & 0.0319 \\
\hline $\begin{array}{c}\text { Difference between maximum and } \\
\text { minimum equivalent strain }\end{array}$ & $8.93 \%$ & $10.36 \%$ & $10.89 \%$ & $11.63 \%$ \\
\hline
\end{tabular}

\section{B. Homogeneous and statistical distribution of crosslinking degree}

At the microscale of the self-sensing smart polymer, the crosslinking degrees are statistically distributed. This is also the limitation of the traditional FE model, which is not capable of representing the crosslinked microstructure, thereby serving as motivation for the network model presented here. To represent the statistical distribution of crosslinking degrees at the microscale, a statistical network model is developed, as shown in figure 8(a). To validate the microstructural representation capability of the statistical network model, a homogeneous network model is constructed and compared with the statistical network model. All crosslinking degrees in the homogeneous network model are kept the same, as shown in figure 8(b). For both the statistical and the homogeneous network models, the same size and geometry as the model used in section III.A $(10 \times 10$ subzones in $1 \mathrm{~mm} \times 1 \mathrm{~mm})$ is maintained. As shown in figure 8(a) and figure 8(b), the numbers in the variable subzones represent the corresponding local crosslinking degrees (unit: \%). The weight percentage of the variable crosslinking degrees in the statistical network model is governed by the probability density function (PDF) obtained from the MD simulations (see figure 3). More details about the PDF of the crosslinking degrees can be found in the authors' previous work. ${ }^{[30]}$ For the homogeneous network model, uniform crosslinking degree, which is equal to the averaged value of all crosslinking degrees in the statistical network model, $(52.72 \%)$ is used for all subzones.

\begin{tabular}{|l|l|l|l|l|l|l|l|l|l|}
\hline 47 & 53 & 50 & 53 & 59 & 50 & 56 & 53 & 56 & 50 \\
\hline 53 & 56 & 59 & 47 & 53 & 56 & 47 & 53 & 50 & 56 \\
\hline 50 & 53 & 50 & 53 & 50 & 53 & 50 & 56 & 47 & 53 \\
\hline 50 & 56 & 53 & 41 & 53 & 44 & 53 & 50 & 53 & 50 \\
\hline 53 & 50 & 62 & 53 & 56 & 50 & 56 & 53 & 56 & 59 \\
\hline 56 & 59 & 47 & 50 & 53 & 56 & 53 & 47 & 50 & 56 \\
\hline 59 & 47 & 56 & 59 & 50 & 59 & 56 & 50 & 53 & 44 \\
\hline 53 & 56 & 50 & 53 & 65 & 44 & 53 & 62 & 50 & 62 \\
\hline 50 & 53 & 53 & 56 & 50 & 53 & 53 & 53 & 56 & 53 \\
\hline 53 & 47 & 50 & 59 & 53 & 56 & 59 & 47 & 53 & 50 \\
\hline
\end{tabular}

a)

\begin{tabular}{|l|l|l|l|l|l|l|l|l|l|}
\hline 53 & 53 & 53 & 53 & 53 & 53 & 53 & 53 & 53 & 53 \\
\hline 53 & 53 & 53 & 53 & 53 & 53 & 53 & 53 & 53 & 53 \\
\hline 53 & 53 & 53 & 53 & 53 & 53 & 53 & 53 & 53 & 53 \\
\hline 53 & 53 & 53 & 53 & 53 & 53 & 53 & 53 & 53 & 53 \\
\hline 53 & 53 & 53 & 53 & 53 & 53 & 53 & 53 & 53 & 53 \\
\hline 53 & 53 & 53 & 53 & 53 & 53 & 53 & 53 & 53 & 53 \\
\hline 53 & 53 & 53 & 53 & 53 & 53 & 53 & 53 & 53 & 53 \\
\hline 53 & 53 & 53 & 53 & 53 & 53 & 53 & 53 & 53 & 53 \\
\hline 53 & 53 & 53 & 53 & 53 & 53 & 53 & 53 & 53 & 53 \\
\hline 53 & 53 & 53 & 53 & 53 & 53 & 53 & 53 & 53 & 53 \\
\hline
\end{tabular}

b)

Figure 8. Crosslinking degree distribution in a) statistical network model and b) homogeneous network model (unit: \%).

Uniaxial tensile test is performed in both statistical and homogeneous network models. The loading is strain control and the maximum loading strain is 5\%. The simulation results are shown in figure 9(a) and figure 9(b), which provide the equivalent strain distribution in the statistical and homogeneous network models, respectively. Results of the average, maximum and minimum equivalent strain in the statistical and homogeneous network models, are presented in Table 1.

As shown in Table 3, the average values of equivalent strain in the statistical network model and the homogeneous network model are close. This implies that the overall mechanical response of the statistical network model corresponds well with the homogeneous model under monotonic loading. The difference between the 
maximum and the minimum strain for statistical network model $(10.36 \%)$ is greater than the corresponding value for homogeneous network model $(8.40 \%)$. Based on the simulation results, a similar conclusion is obtained, as in section III.A. The statistical distribution of crosslinking degrees results in a larger distinguishable disorder in strain distribution indicating a high strain gradient. Consequently, compared with the homogeneous microstructure the statistical microstructure can lead to a higher damage initiation.

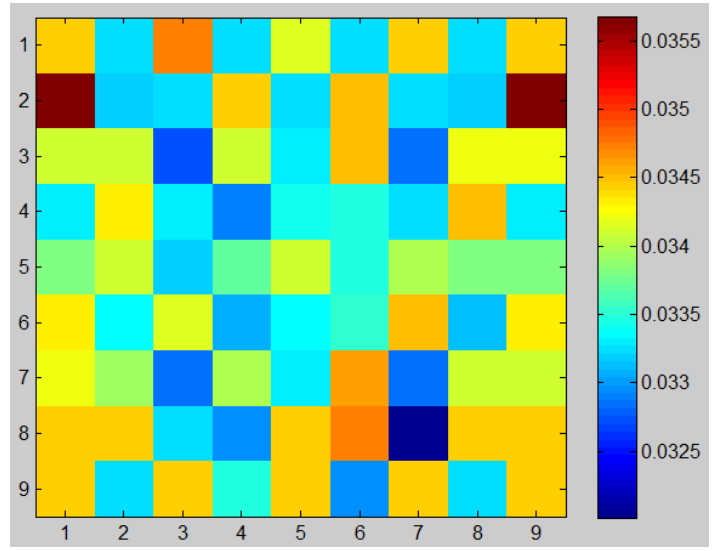

a)

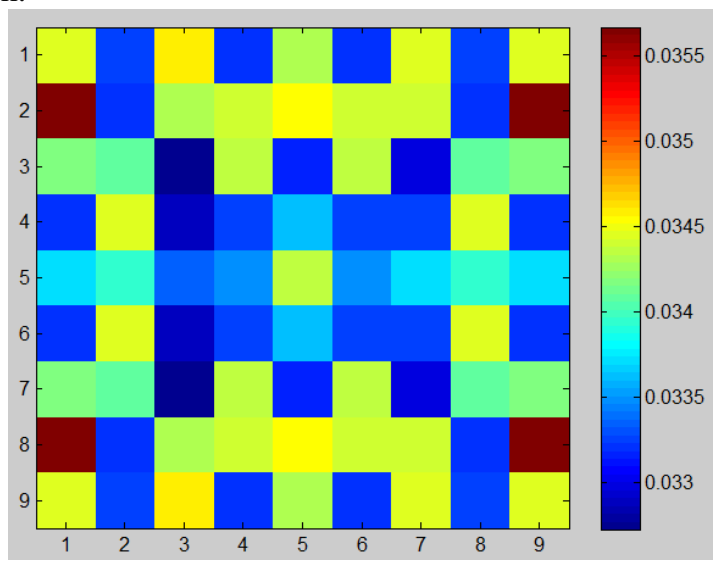

b)

Figure 9. Equivalent strain distribution in a) statistical network model and b) homogeneous network model.

Table 3. Equivalent strain in statistical and homogeneous network models

\begin{tabular}{|c|c|c|}
\hline & Statistical & Homogeneous \\
\hline Average equivalent strain & 0.0338 & 0.0339 \\
\hline Maximum equivalent strain & 0.0357 & 0.0357 \\
\hline Minimum equivalent strain & 0.0320 & 0.0327 \\
\hline $\begin{array}{c}\text { Difference between maximum and } \\
\text { minimum equivalent strain }\end{array}$ & $10.36 \%$ & $8.40 \%$ \\
\hline
\end{tabular}

\section{Effect of spring length in network model}

As discussed earlier, the two steps in developing a network model are: i) designing the configuration variables including arrangement, spring size, and spring stiffness; ii) bridging MD simulation and network model using mechanical equivalence optimization. In the first step, the definition of spring stiffness is related to the spring length. In the second step, the mechanical equivalence provides the network model with the most proximate mechanical response of the MD simulation. Technically, the spring length has a negligible effect on the mechanical response of the network model due to the mechanical equivalence optimization conducted in the second step of the development of the network model. To understand the spring length effect on the material behavior representation, two network models with different spring lengths were generated. The sizes of the two network models were $8 \mu \mathrm{m} \times 8 \mu \mathrm{m}$ and 1 $\mathrm{mm} \times 1 \mathrm{~mm}$ respectively, and the corresponding spring lengths in the two network models were $0.8 \mu \mathrm{m}$ and $0.1 \mathrm{~mm}$ respectively. Based on the simulation results shown in Table 4, the material characteristics including tensile modulus, compressive modulus and shear modulus represented by the two network models are close (error is less than $1 \%$ ), validating the hypothesis that there is negligible effect of spring length on the mechanical response of the network model. However, shorter spring lengths can capture mechanical responses at shorter length scales, and thereby enhance simulation accuracy. Additionally, corresponding computational efficiency is reduced because of the increased number of springs. Spring length, therefore, plays a similar role as that of mesh size in an FE model.

Table 4. Mechanical properties in network models of different spring lengths.

\begin{tabular}{|c|c|c|c|}
\hline & Tensile Modulus & Compressive Modulus & Shear Modulus \\
\hline $\begin{array}{c}\text { Network model of short } \\
\text { springs }(0.8 \mu \mathrm{m})\end{array}$ & $1.778 \mathrm{GPa}$ & $1.752 \mathrm{GPa}$ & $0.502 \mathrm{GPa}$ \\
\hline Network model of long & $1.769 \mathrm{GPa}$ & $1.744 \mathrm{GPa}$ & $0.503 \mathrm{GPa}$ \\
\hline
\end{tabular}




\begin{tabular}{|c|c|c|c|}
\hline springs $(0.1 \mathrm{~mm})$ & & & \\
\hline $\begin{array}{c}\text { Error of mechanical } \\
\text { properties }\end{array}$ & $0.51 \%$ & $0.46 \%$ & $0.20 \%$ \\
\hline
\end{tabular}

\section{Multiscale integration based on a statistical network model}

\section{A. Reliability of statistical network model}

A set of three meso statistical network models were constructed to validate the simulation accuracy (see figure 10(b), figure 10(c) and figure 10(d)). For each the three network models, the same size and geometry as the model used in section III.A $(10 \times 10$ subzones in $1 \mathrm{~mm} \times 1 \mathrm{~mm})$ is maintained. There are 121 beads, 220 short springs, and 200 long springs in the 8 neighbors based network model. The lengths of the short and long springs in the network models are $0.1 \mathrm{~mm}$ and $0.1414 \mathrm{~mm}$, respectively. The numbers shown in the square subzones in figure 10(b), figure $10(\mathrm{c})$, and figure 10(d) represent the local crosslinking degrees.

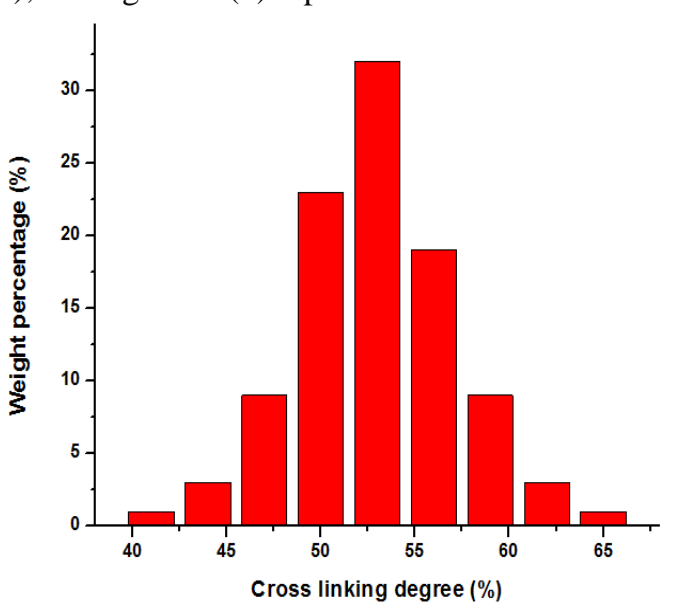

a)

\begin{tabular}{|l|l|l|l|l|l|l|l|l|l|}
\hline 47 & 56 & 50 & 53 & 56 & 53 & 50 & 59 & 56 & 47 \\
\hline 53 & 50 & 53 & 53 & 47 & 50 & 53 & 53 & 53 & 56 \\
\hline 53 & 56 & 47 & 50 & 53 & 59 & 44 & 50 & 50 & 62 \\
\hline 47 & 50 & 56 & 53 & 50 & 56 & 56 & 53 & 44 & 53 \\
\hline 53 & 53 & 59 & 50 & 53 & 53 & 50 & 53 & 50 & 59 \\
\hline 50 & 56 & 47 & 53 & 50 & 47 & 56 & 53 & 56 & 53 \\
\hline 59 & 41 & 59 & 53 & 56 & 62 & 53 & 56 & 59 & 50 \\
\hline 50 & 56 & 50 & 53 & 56 & 53 & 50 & 56 & 53 & 53 \\
\hline 62 & 50 & 53 & 56 & 44 & 50 & 53 & 56 & 53 & 65 \\
\hline 47 & 59 & 53 & 50 & 56 & 53 & 50 & 50 & 47 & 59 \\
\hline
\end{tabular}

c)

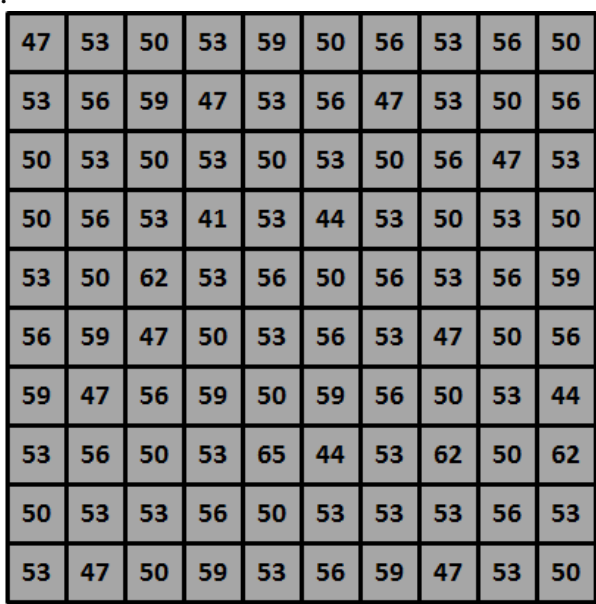

b)

\begin{tabular}{|l|l|l|l|l|l|l|l|l|l|}
\hline 50 & 53 & 53 & 50 & 53 & 56 & 50 & 59 & 50 & 47 \\
\hline 53 & 44 & 56 & 53 & 47 & 59 & 53 & 56 & 53 & 53 \\
\hline 50 & 56 & 53 & 56 & 50 & 53 & 56 & 50 & 50 & 59 \\
\hline 56 & 53 & 56 & 50 & 53 & 53 & 50 & 47 & 56 & 53 \\
\hline 50 & 53 & 47 & 53 & 56 & 50 & 59 & 53 & 56 & 50 \\
\hline 47 & 50 & 53 & 59 & 53 & 41 & 56 & 50 & 50 & 47 \\
\hline 53 & 53 & 59 & 56 & 44 & 62 & 50 & 53 & 59 & 53 \\
\hline 56 & 50 & 50 & 53 & 50 & 53 & 59 & 53 & 56 & 62 \\
\hline 53 & 53 & 47 & 56 & 53 & 56 & 47 & 53 & 50 & 44 \\
\hline 50 & 59 & 56 & 53 & 62 & 50 & 53 & 65 & 56 & 47 \\
\hline
\end{tabular}

d)

Figure 10. a) Probability distribution of crosslinking degree in the statistical network model, b) network model 1 c) network model 2 , and d) network model 3.

Simulations were conducted using uniaxial strain controlled tensile load; the maximum loading strain was $10 \%$. The average equivalent strain in the three network models was calculated. It was observed that the average equivalent strain in the three statistical network models was very close and the error was less than $1 \%$ (see figure 11). The results establish the validity and robustness of the representative models. It should be noted that the spring- 
bead based network model improves the computational efficiency significantly. For a similar uniaxial tensile loading simulation 20 hours of runtime is required using MD modeling whereas a network model completes the simulation in 4 minutes. The simulation efficiency is improved by 300 times using the network model.

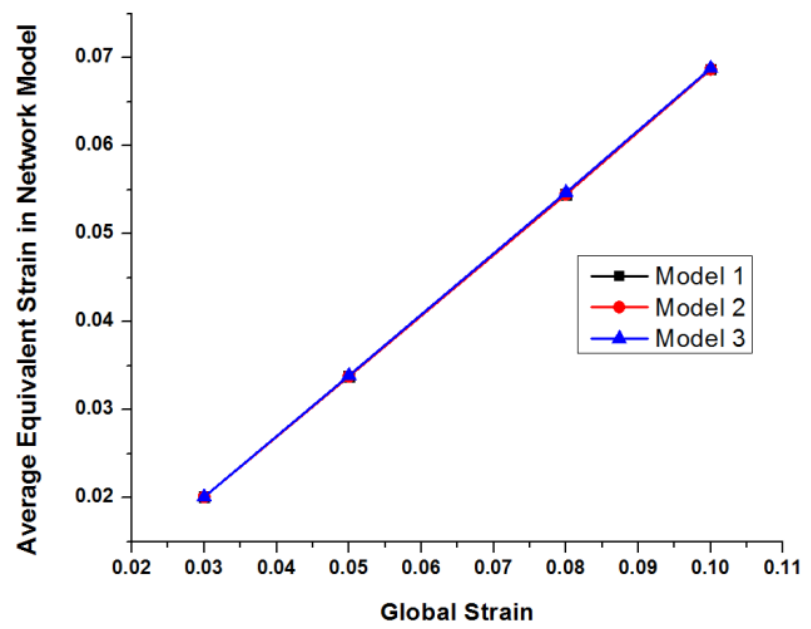

Figure 11. Average equivalent strain in three statistical network models vs. loading strain.

\section{B. Local strain and crosslinking degree in the network model}

Simulations were conducted with the statistical network model to study the relation between local strain and crosslinking degree using biaxial strain controlled tensile load; the maximum loading strain was $10 \%$ in both directions. Results are presented for the statistical model 1, shown in figure 12(a). The distribution of equivalent strain is shown in figure 12(b). There is an obvious relation between local strain and crosslinking degree: high strain concentration (the red band in figure 12(b)) occurs mostly where there is low crosslinking degree (the red square zone in figure 12(a)) especially where the crosslinking degree is below $52.72 \%$ (average crosslinking degree in the model). These results are physically meaningful; lower material stiffness leads to a higher strain under the same external loads. The locations with high strain concentration also correlate to potential local defects. The damage evaluation based on MD \& network model simulations will be discussed in section V.

\begin{tabular}{|l|l|l|l|l|l|l|l|l|l|}
\hline 47 & 53 & 50 & 53 & 59 & 50 & 56 & 53 & 56 & 50 \\
\hline 53 & 56 & 59 & 47 & 53 & 56 & 47 & 53 & 50 & 56 \\
\hline 50 & 53 & 50 & 53 & 50 & 53 & 50 & 56 & 47 & 53 \\
\hline 50 & 56 & 53 & 41 & 53 & 44 & 53 & 50 & 53 & 50 \\
\hline 53 & 50 & 62 & 53 & 56 & 50 & 56 & 53 & 56 & 59 \\
\hline 56 & 59 & 47 & 50 & 53 & 56 & 53 & 47 & 50 & 56 \\
\hline 59 & 47 & 56 & 59 & 50 & 59 & 56 & 50 & 53 & 44 \\
\hline 53 & 56 & 50 & 53 & 65 & 44 & 53 & 62 & 50 & 62 \\
\hline 50 & 53 & 53 & 56 & 50 & 53 & 53 & 53 & 56 & 53 \\
\hline 53 & 47 & 50 & 59 & 53 & 56 & 59 & 47 & 53 & 50 \\
\hline
\end{tabular}

a)

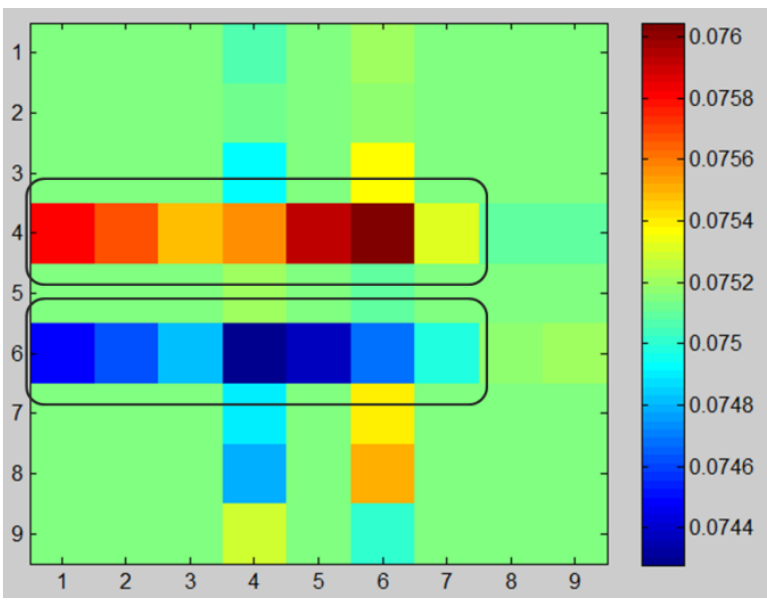

b)

Figure 12. Simulation of statistical network model under biaxial tensile load: a) crosslinking degree distribution in network model and b) distribution of equivalent strain in network model.

\section{Comparison between network model and FE model}

The spring-bead based network model was developed in order to represent the smart polymer at the mesoscale. To capture the material behavior from mesoscale to macroscale, a coupled network and an FE model are required. The meso network model is explicitly modeled and embedded at the hot spot within a FE mesh of the structure so 
that different length scales can be bridged. A notched dog bone specimen was designed for simulation under uniaxial tensile loads. The dimensions of the notched dog bone specimen are shown in figure 13. The network model and FE model were used concurrently to represent the material properties at the notch tip and the remaining section, respectively (see figure 14(a)). An FE model without embedded springs and beads was developed (see figure 14(b)). To validate the network model based multiscale modeling approach two measures were considered: hot spot location and far field distributions. To ensure the network model is representative of the bulk material and mechanical responses both measures must remain consistent with or without a network model implemented in the FE model.

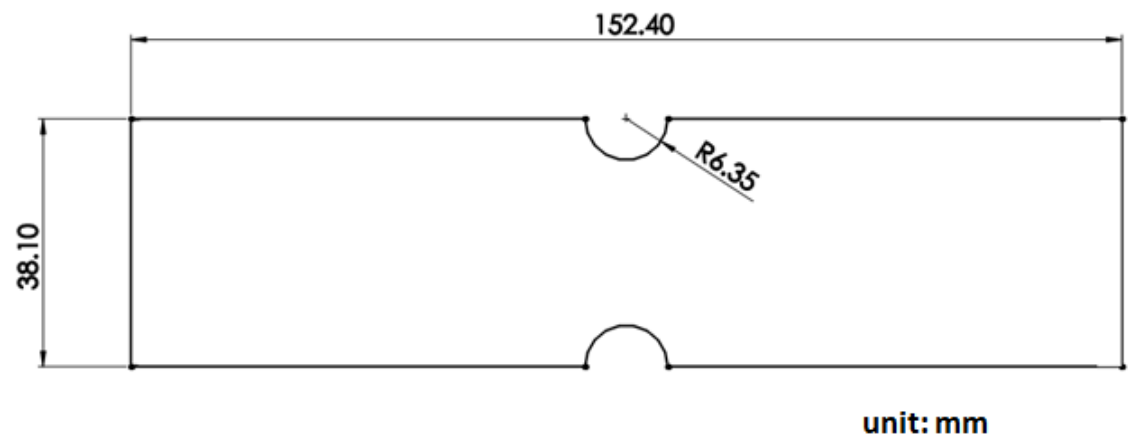

Figure 13. Dimensional drawing of dog bone specimen for simulation.

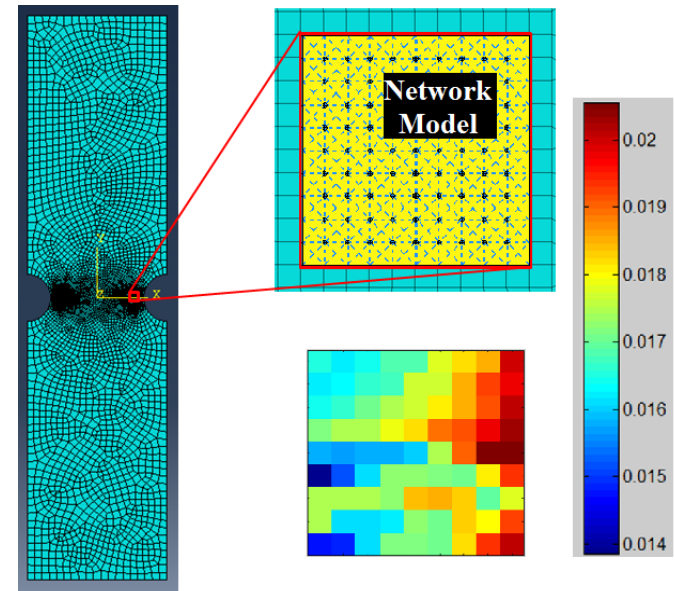

a)

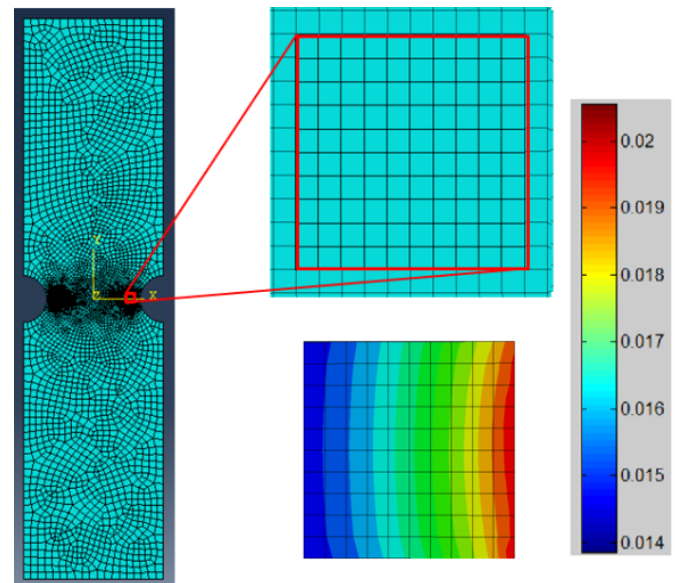

b)

Figure 14. Strain distribution in the hot spot using a) network model and b) FE model.

Comparison of the strain distributions obtained using the two models are shown in figure 14. In both, the network model (figure 14(a)) and FE model (figure 14(b)), the high strain concentrations are observed near the notch tip. However, the strain distribution in the network model shows larger variability due to the variability in the crosslinking degree. The local strain distribution obtained using the network model is expected to provide a more accurate estimate of the local defects at the microscale. figure 15 shows the comparison of strain distributions outside the hot spots in the simulations, with and without a network model. A good correlation can be observed between the network model and the FE model, validating the accuracy of the network model with the FE model outside of the hot spot (notch tip). 


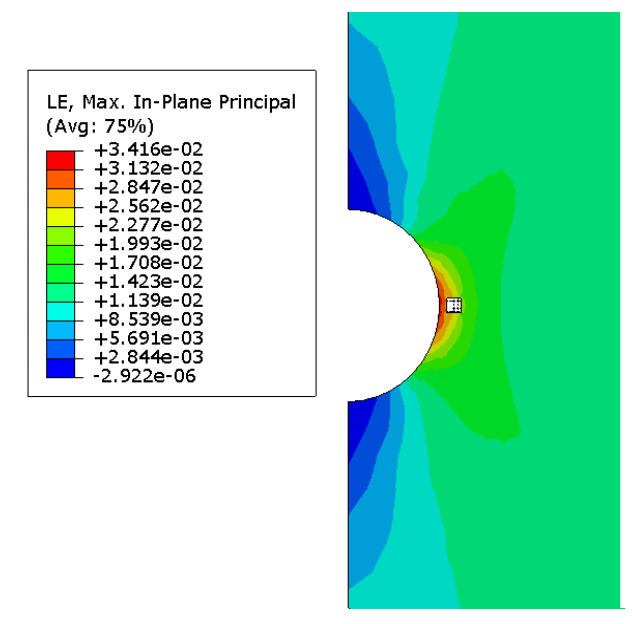

a)

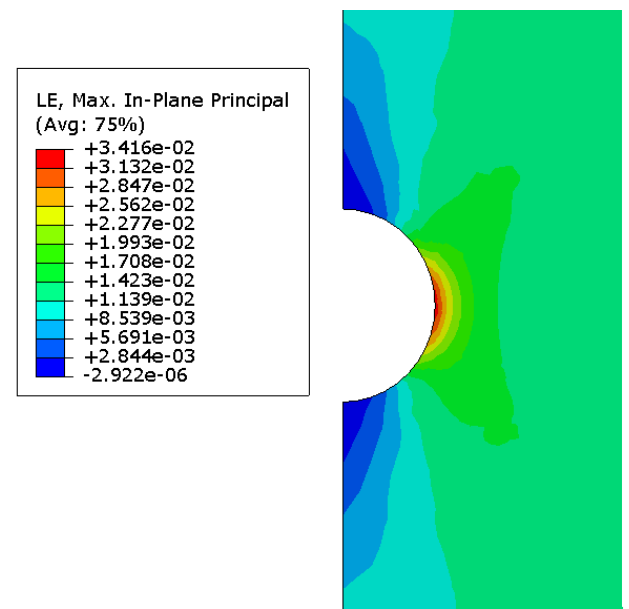

b)

Figure 15. Strain distribution within notched specimen a) with network model and b) without network model.

\section{Damage estimation using statistical network model}

\section{A. Evaluation of self-sensing intensity of a smart polymer}

In this research TCEs were used as the stress-sensing material to detect the defects in the smart polymer. The epoxy resin is brittle with a propensity to crack if exposed to sufficient deformation, leading to irreversible damage in the form of micro cracking. In the high strain concentration locations of the material the force can be transferred through the bulk material to individual polymer chains, and then to the cleavable bonds on the mechanophore units. The local deformation in these regions exceeds the critical limitation of the polymer chains and results in the permanent deformation under rupture of crosslinked bonds. ${ }^{[4]}$ This early damage precursor can be detected through the employment of TCE-containing polymers. Note that the fluorescence response of the mechanophore results from the rupture of the covalent bonds in cyclobutane. It is also related to the onset of permanent strain (deformation) of the bonds at the microscale. The fluorescence intensity is also directly dependent on the density of the breaking bonds. The novel spring-bead based network model representing this TCE embedded polymer is also required to simulate this self-sensing feature. Therefore, there is a correlation between the local strain and the color changing phenomena. This relation between fluorescence intensity in the experiments and the mechanical response in the simulation using the network model was studied.

Uniaxial compressive tests were performed to obtain the fluorescence intensities of the smart material under different loads. A Test Resources Electromechanical System (with the maximum loading capability $4.4 \mathrm{kN}$ ) was used to conduct the compressive tests; stress-strain curves were also obtained. The tests were performed with displacement control in a longitudinal direction at a loading rate of $1 \mathrm{~mm} / \mathrm{min}$. The maximum loading strain was $10 \%$ to ensure that the material deformation remained in the elastic range. To monitor the fluorescence from defects in the polymer, the cubic samples of polymer blends were compressed to different strains and observed under the fluorescence microscope (Nikon Elipse TE300 inverted video microscope) by exposure to 330-380 nm UV light. An image processing program, ImageJ, was used to process the fluorescent micrographs and capture the fluorescence intensities. The micrographs were taken at the same location of the specimen surface, with the size of $427 \mu \mathrm{m} \times 325$ $\mu \mathrm{m}$. The integrated fluorescence intensity was calculated through the summation of all pixel values in the micrograph. A quasi-linear relation was observed between the fluorescence intensity density and the loading strain (see figure 16(a)). Figure 16(b) and figure16(c) show the microscopic images of the specimen surface and the corresponding fluorescence responses when the loading strain is $4 \%$ and $6 \%$, respectively. The relation between fluorescence intensity density and the loading strain can be described using a linear fit:

$$
I=117.35 \varepsilon+123.17
$$


where $\boldsymbol{I}$ represents the observed fluorescence intensity density, and $\boldsymbol{\varepsilon}$ is the loading strain.

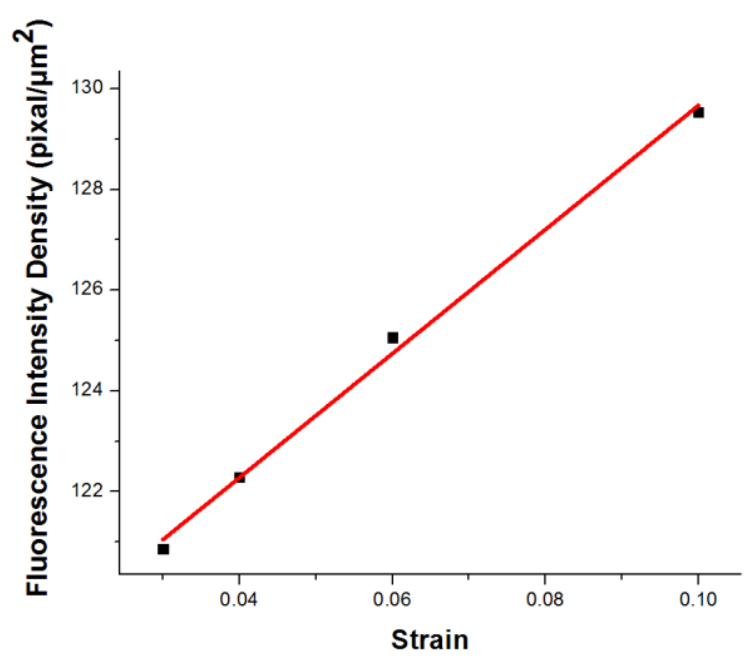

a)

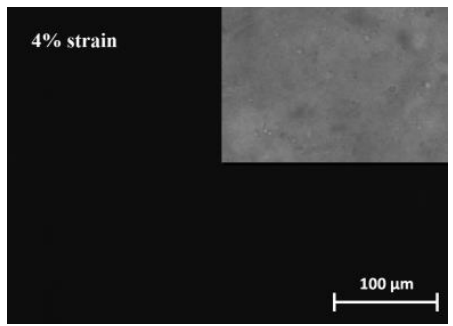

b)

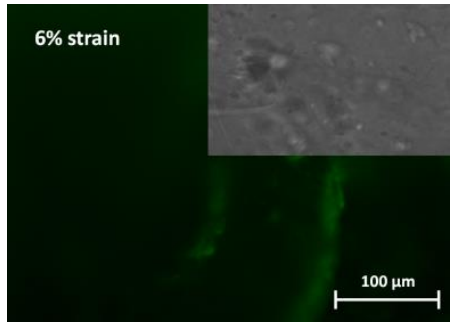

c)

Figure 16. Fluorescence observation of self-sensing smart polymer. a) Integrated intensity density vs. loading strain in fluorescence observation, b) Microscopic images and detected fluorescence of specimen at $4 \%$ strain, and c) Microscopic images and detected fluorescence of specimen at $6 \%$ strain.

To capture the self-sensing performance, simulation was performed using the statistical network model 1 , as shown in figure 10(b). Uniaxial compressive loads of the same magnitude as that of experiments were applied to the network model. The equivalent strain in the network model was calculated, which shows a quasi-linear relation between the average equivalent strain and the loading strain (figure 17(a)). The distribution of equivalent strain in the network model under different loading strain can be seen in figure 17(b)-(e). The simulation result shows that the strain distribution is influenced by the local crosslinking degree. Also, both local and average equivalent strain increases linearly with loading. A linear fit is used to describe the relation between average equivalent strain and the global loading strain:

$$
\bar{\varepsilon}=0.694 \varepsilon-0.00081
$$

where $\boldsymbol{\varepsilon}$ is the loading strain, and $\bar{\varepsilon}$ is the average equivalent strain in the statistical network model. As shown in Eqs. 4 and 5, both the fluorescence intensity and the strain in the network model exhibit a quasi-linear relationship with respect to the loading strain (see figure 16(a) and figure 17(a)). A function describing the relation between average strain $\overline{\boldsymbol{\varepsilon}}$ in the network model and fluorescence intensity density $\boldsymbol{I}$ from experimental observation can be derived by combining Eqs. (4) and (5):

$$
\boldsymbol{I}=169.09 \bar{\varepsilon}+123.17
$$

Thus, the average equivalent strain from the network model based simulation can be used to describe the observed fluorescence intensity of the TCE-embedded polymer.

These results indicate that the average equivalent strain obtained from the network model can be used as an index to describe the self-sensing fluorescence intensity. This approach of evaluating fluorescence intensity numerically using the high efficiency network model can reduce the experimental cost significantly. 


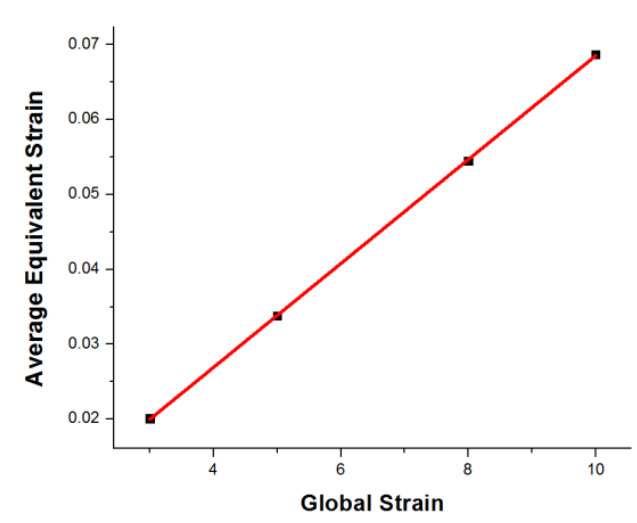

a)

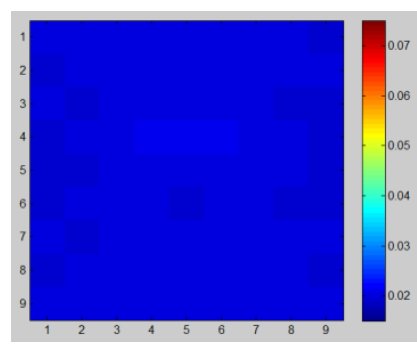

b)

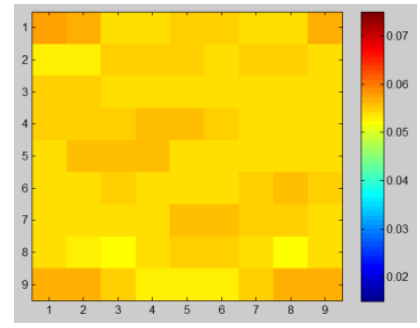

d)

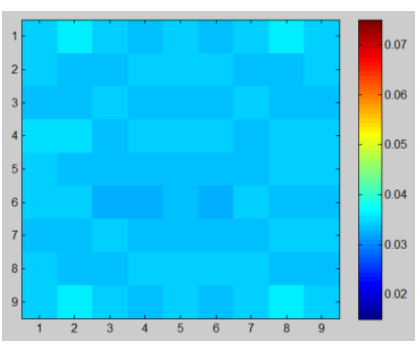

c)

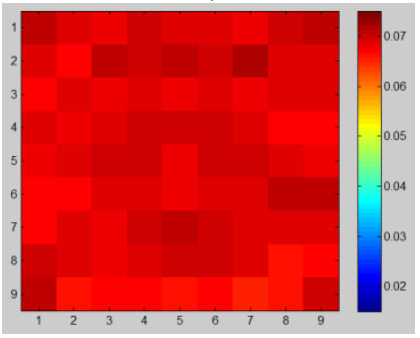

e)

Figure 17. Network model based simulation results: a) Average equivalent strain in network model; and equivalent strain distribution at different global strain at b) $3 \%$, c) $5 \%$, d) $8 \%$, and e) $10 \%$.

\section{B. Strain based damage evaluation to predict failure}

In the MD simulation uniaxial tensile tests were implemented on a series of RVE models with different crosslinking degrees. The RVE size, molecular constituents and MD simulation conditions have been discussed in section II. The simulation results showed that the stress (load) in the RVE model increased in a fluctuating curve with strain (see figure 18). One important conclusion from the MD results is that the first significant kink of the stress-strain curves takes place where the strain value is around $8 \%$ for a range of crosslinking degrees. This phenomenon indicates that there is a notable microstructural defect resulting from the rupture of polymer chains at the kink point. The relationship between the mechanical response at the kink point and the damage initiation has also been observed in experiments and simulations conducted by other researchers. Kingsbury et al. obtained the threshold kink point of the stress-strain curve of mechanophore crosslinked polymer in their experiments. ${ }^{4}$ Their results also corresponded with the onset of the mechanochemical activation in the fluorescence observation. Silberstein et al. found a non-equilibrium state resulting in local force fluctuations in glassy polymers. ${ }^{5}$ The MD simulation results showed good agreement between the onset of strain softening (first kink) and the onset of mechanophore activation.

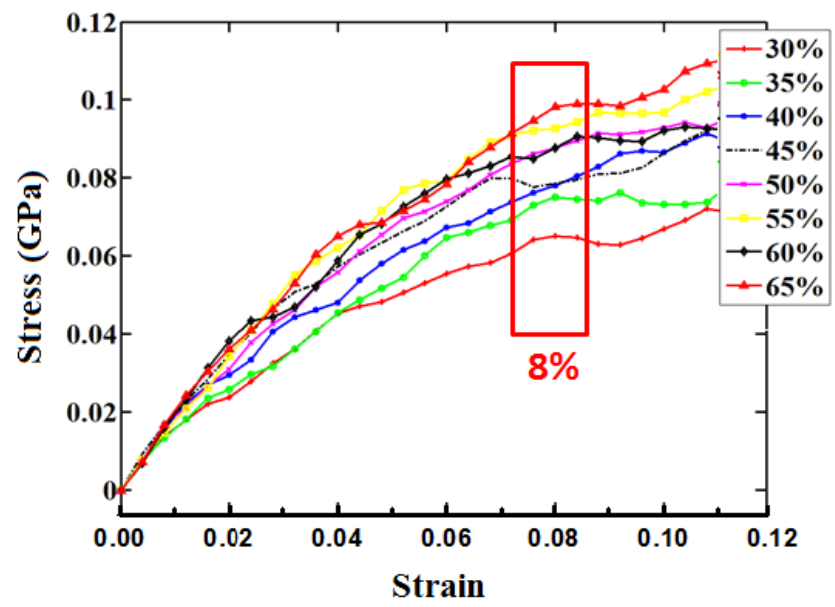

Figure 18. Tensile mechanical responses of smart polymer at variable crosslinking degrees based on the MD simulation. 
Based on the damage analysis in the MD simulation, there are micro cracks when the local strain is $8 \%$, associated with the observed initial kink point of the stress-strain curve. Next, simulations were conducted using the network model to observe the strain evolution. Statistical network model 1 (see figure 10(b)) was used under a uniaxial tensile load. The equivalent strain distribution from the simulation results is shown in figure 19. It is observed that the local equivalent strain increases with loading. The loading strain implemented on the network model is $11.2 \%$, when the maximum local equivalent strain exceeds $8 \%$, indicating permanent tensile strain for this smart material.

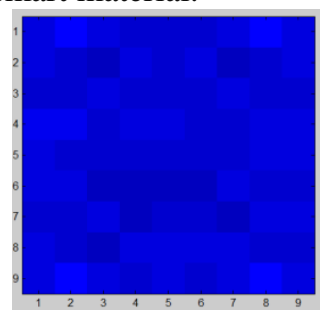

a)

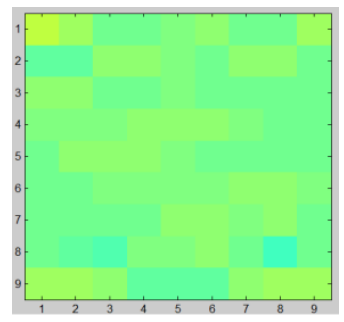

b)

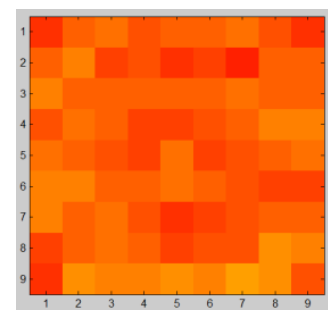

c)

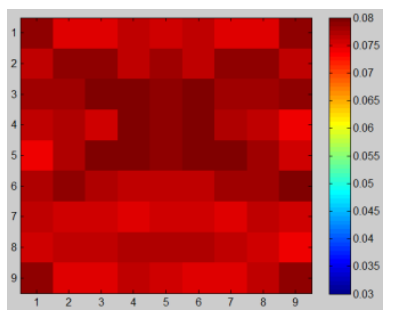

d)

Figure 19. Equivalent strain distribution in statistical network model 1 at different loading strain: a) $5 \%$, b) $8 \%$, c) $10 \%$, and d) $11 \%$.

Experiments were conducted to validate the damage evaluation phenomenon of the smart polymer. A set of three uniaxial tensile tests were conducted. The Test Resources Electromechanical System (with the maximum loading capability $4.4 \mathrm{kN}$ ) was also used to conduct the tensile tests. From the experimental results the material showed elastic behavior until failure (see figure 20). The average permanent tensile strain was $10.6 \%$ based on experimental observation. The error of the permanent tensile strain between experiment and simulation was 5.36\%. This statistical network model, therefore, was capable of evaluating the damage initiation in the polymer material.

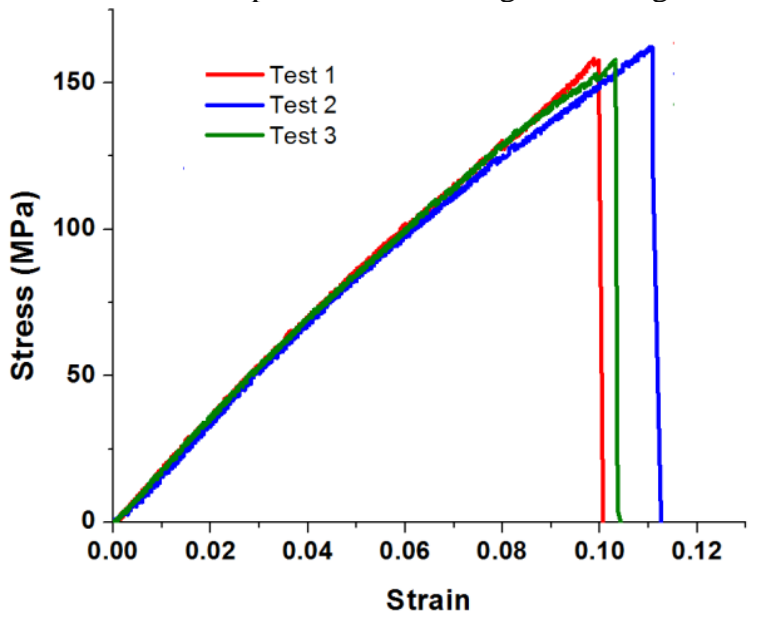

Figure 20. Experimental results of tensile tests.

\section{Conclusion}

A multiscale approach is developed here to model the mechanical properties of a novel mechanophore crosslinked polymer that is capable of detecting damage precursor. A spring-bead based network model is developed to represent the self-sensing smart polymeric material. Three different length scales are considered. At the microscale, the spring-bead model is constructed based on the MD simulation results to represent crosslinked polymer chains. At the mesoscale, a network model is developed to capture the statistical material properties. At the macroscale, a coupled network and the FE model is constructed to represent the mechanical response in the structure. A series of parametric studies are performed to understand the microstructural behavior of the smart material. The results show that the local crosslinking degree has a significant effect on the mechanical properties of the material. A high crosslinking degree value leads to low local strain, which demonstrates stronger material 
performance. A larger difference between neighbor crosslinking degrees can increase the strain gradient, which then leads to higher local defects. Results from the simulations and experiments were combined to establish a correlation between the mechanophore fluorescence and local strain. A linear relation between average strain in the network model and fluorescence intensity density in the experimental observation is also obtained. The computationally efficient statistical network model is seen as capable of describing the self-sensing phenomenon using the relationship between local equivalent strain in the network model and the observed fluorescence intensity. The statistical network model is also used to estimate the damage initiation by analyzing the MD and network simulation results. Good agreement was observed between simulation and experiment. Although the thermal and chemical properties of the mechanophore are not addressed in the network model to improve simulation efficiency, this study shows that the statistical network model can successfully capture the dynamic behavior of the self-sensing smart polymer material.

\section{Acknowledgements}

This work is partially supported by the Air Force Office of Scientific Research (Grant number: FA9550-12-1-0331), and the program manager is Dr. David Stargel. This work is also partially supported by the Office of Naval Research (Grant number: N00014-14-1-0068), and the program manager is Dr. William Nickerson.

\section{References}

${ }^{1}$ Thostenson, E. T., and Chou T. W. "Carbon nanotube networks: sensing of distributed strain and damage for life prediction and self healing," Advanced Materials, Vol.18, No.21, 2006, pp. 2837-2841.

${ }^{2}$ Wu, D. Y., Meure S., and Solomon D. "Self-healing polymeric materials: a review of recent developments," Progress in Polymer Science, Vol. 33, No.5, 2008, pp. 479-522.

${ }^{3}$ Black, A. L., Lenhardt, J. M., and Craig, S. L., "From molecular mechanochemistry to stress-responsive materials." Journal of Materials Chemistry, Vol. 21, No. 6, 2011, pp. 1655-1663.

${ }^{4}$ Kingsbury, C. M., et al. "Shear activation of mechanophore-crosslinked polymers," Journal of Materials Chemistry, Vol. 21, No.23, 2011, pp. 8381-8388.

${ }^{5}$ Silberstein, M. N., et al. "Modeling mechanophore activation within a crosslinked glassy matrix," Journal of Applied Physics, Vol. 114, No.2, 2013, pp. 023504.

${ }^{6}$ Cho, S. Y., Kim J. G., and Chung C. M., "A fluorescent crack sensor based on cyclobutane-containing crosslinked polymers of tricinnamates," Sensors and Actuators B: Chemical, Vol. 134, No. 2, 2008 , pp. 822-825.

${ }^{7}$ Cho, S. Y., Kim J. G., and Chung C. M., "Photochemical Crack Healing in Cinnamate based Polymers," Journal of Nanoscience and Nanotechnology, Vol. 10, No. 10, 2010, pp. 6972-6976.

${ }^{8}$ Zou, J., et al. "Early damage detection in epoxy matrix using cyclobutane-based polymers," Smart Materials and Structures, Vol. 23, No.9, 2014, pp. 095038.

${ }^{9}$ Flory P. J., and Rehner Jr. J., "Statistical Mechanics of Cross-Linked Polymer Networks II. Swelling," The Journal of Chemical Physics, Vol. 11, No.11, 1943, pp. 521-526.

${ }^{10}$ Krumova M., et al. "Effect of crosslinking on the mechanical and thermal properties of poly (vinyl alcohol)," Polymer, Vol. 41, No.26, 2000, pp. 9265-9272.

${ }^{11}$ Fan H. B., and Yuen M. M., "Material properties of the crosslinked epoxy resin compound predicted by molecular dynamics simulation," Polymer, Vol. 48, No.7, 2007, pp. 2174-2178.

${ }^{12}$ Ghosh, S., Lee, K., Moorthy, S., "Multiple scale analysis of heterogeneous elastic structures using homogenization theory and voronoi cell finite element method," Int. Journal of Solids and Structures, Vol. 32, No. 1, 1995, pp. 27-62.

${ }^{13}$ Borodin O., et al, "Multiscale modeling of viscoelastic properties of polymer nanocomposites," Journal of Polymer Science Part B: Polymer Physics, Vol. 43, No. 8, 2005, pp. 1005-1013.

${ }^{14}$ Fermeglia M., and Pricl S., "Multiscale modeling for polymer systems of industrial interest," Progress in organic coatings, Vol. 58, No.2, 2007, pp. 187-199.

${ }^{15}$ Yarovsky I., and Evans E., "Computer simulation of structure and properties of crosslinked polymers: application to epoxy resins," Polymer, Vol. 43, No.3, 2002, pp. 963-969.

${ }^{16}$ Neerukatti, R. K., Liu K.C., Kovvali N. and Chattopadhyay A., "Fatigue life prediction using hybrid prognosis for structural health monitoring," Journal of Aerospace Information Systems, Vol. 11, No. 4, 2014, pp. $211-232$.

${ }^{17}$ Peng, T., et al., "A novel Bayesian imaging method for probabilistic delamination detection of composite materials,” Smart Materials and Structures, Vol. 22, No.12, 2013, pp. 125019. 
${ }^{18}$ Peng, T., et al., "Integrated experimental and numerical investigation for fatigue damage diagnosis in
composite plates," Structural Health Monitoring, $2014,1475921714532992$.
${ }^{19}$ Onck, P. R., et al., "Alternative explanation of stiffening in crosslinked semiflexible networks," Physical
review letters, Vol. 95, No.17, 2005, pp. 178102 .
${ }^{20}$ Jiang, H., et al., "Finite element method parametric study on scratch behavior of polymers," Journal of
Polymer Science Part B: Polymer Physics, Vol. 45 , No.12, 2007 , pp. 1435-1447.
${ }^{21}$ Chen, Y., and Dimitris C. L., "A constitutive theory for shape memory polymers. Part I: Large
deformations," Journal of the Mechanics and Physics of Solids, Vol. 56, No.5, 2008, pp. 1752-1765.
${ }^{22}$ Westbrook, K. K., et al., "A 3D finite deformation constitutive model for amorphous shape memory polymers: a multi-branch modeling approach for nonequilibrium relaxation processes," Mechanics of Materials, Vol. 43, No. 12, 2011, pp. 853-869.

${ }^{23}$ Volk, B. L., Lagoudas, D. C., and Chen, Y. "Analysis of the finite deformation response of shape memory polymers: II. 1D calibration and numerical implementation of a finite deformation, thermoelastic model," Smart Materials and Structures, Vol. 19, No. 7, 2010, pp. 075006.

${ }^{24}$ Diani, J., et al. "Predicting thermal shape memory of crosslinked polymer networks from linear viscoelasticity." International Journal of Solids and Structures 49.5 (2012): 793-799.

${ }^{25}$ Buxton, G. A., and Balazs, A. C., "Lattice spring model of filled polymers and nanocomposites," Journal of chemical physics, Vol. 117, No. 16, 2002, pp. 7649-7658.

${ }^{26}$ Iwata K., "Irreversible Statistical Mechanics of Polymer Chains. I. Fokker-Planck Diffusion Equation," Journal of Chemical Physics, Vol. 54, No. 1, 2003, pp. 12-26.

${ }^{27}$ Schwarz U. S., Erdmann T., and Bischofs I. B., "Focal adhesions as mechanosensors: the two-spring model," Biosystems, Vol. 83, No. 2, 2006, pp. 225-232.

${ }^{28}$ Underhill P. T., and Doyle P. S., "Development of bead-spring polymer models using the constant extension ensemble," Journal of Rheology, Vol. 49, No.5, 2005, pp. 963-987.

${ }^{29}$ Indei, T., Schieber, J. D. and Takimoto, J., "Effects of fluctuations of crosslinking points on viscoelastic properties of associating polymer networks," Rheologica acta, Vol. 51, No.11-12, 2012, pp. 1021-1039.

${ }^{30}$ Zhang, J., Koo, B., Subramanian, N., Liu, Y. and Chattopadhyay, A., "Statistical Multiscale Modeling of Smart Polymer Materials using a Spring-Bead Based Network Model," 55TH AIAA/ASME/ASCE/AHS/SC STRUCTURES, STRUCTURAL DYNAMICS, AND MATERIALS CONFERENCE, 2014, January13-17, National Harbor, Maryland

${ }^{31}$ Halgren, T. A., "Merck molecular force field. I. Basis, form, scope, parameterization, and performance of MMFF94," Journal of computational chemistry, Vol. 17, No.5-6, 1996, pp. 490-519.

${ }^{32}$ Plimpton, S., Crozier, P., and Thompson, A., "LAMMPS-large-scale atomic/molecular massively parallel simulator," Vol. 18, 2007, Sandia National Laboratories.

${ }^{33}$ Espuche, E., et al., "Influence of crosslink density and chain flexibility on mechanical properties of model epoxy networks," Macromolecular symposia, Vol. 93., No. 1., 1995, Hüthig \& Wepf Verlag.

${ }^{34}$ Charulatha, V., and Rajaram, A., "Influence of different crosslinking treatments on the physical properties of collagen membranes," Biomaterials, Vol. 24, No.5, 2003, pp. 759-767.

${ }^{35}$ Park, H. B., et al., "Effect of crosslinked chain length in sulfonated polyimide membranes on water sorption, proton conduction, and methanol permeation properties," Journal of membrane science, Vol. 285, No.1, 2006, pp. 432-443.

${ }^{36}$ Zosel, A., and Ley, G., "Influence of crosslinking on structure, mechanical properties, and strength of latex films," Macromolecules, Vol. 26, No. 9, 1993, pp. 2222-2227.

${ }^{37}$ Halary, J. L., "Structure-property relationships in epoxy-amine networks of well-controlled architecture," High performance polymers, Vol. 12, No.1, 2000, pp. 141-153.

${ }^{38}$ Berger, J., et al., "Structure and interactions in covalently and ionically crosslinked chitosan hydrogels for biomedical applications," European Journal of Pharmaceutics and Biopharmaceutics, Vol. 57, No. 1, 2004, pp. 1934.

${ }^{39}$ Urbaczewski-Espuche, E., et al., "Influence of chain flexibility and crosslink density on mechanical properties of epoxy/amine networks,” Polymer engineering \& science, Vol. 31, No. 22, 1991, pp. 1572-1580.

${ }^{40}$ Svaneborg, C., Grest, G. S., and Everaers, R., "Disorder effects on the strain response of model polymer networks," Polymer, Vol. 46, No. 12, 2005, pp. 4283-4295.

${ }^{41} \mathrm{Li}$, J., et al., "A micromechanics-based strain gradient damage model for fracture prediction of brittle materials-Part II: Damage modeling and numerical simulations," International Journal of Solids and Structures, Vol. 48, No.24, 2011, pp. 3346-3358.

${ }^{42}$ Geers, M. G. D., et al., "Strain-based transient-gradient damage model for failure analyses," Computer Methods in Applied Mechanics and Engineering, Vol. 160, No.1, 1998, pp. 133-153. 
${ }^{43}$ Francfort, Gilles A., and J-J. Marigo. "Revisiting brittle fracture as an energy minimization problem." Journal of the Mechanics and Physics of Solids 46.8 (1998): 1319-1342.

${ }^{44}$ Buehler, Markus J., Sinan Keten, and Theodor Ackbarow. "Theoretical and computational hierarchical nanomechanics of protein materials: Deformation and fracture." Progress in Materials Science 53.8 (2008): 11011241. 